\title{
Evidence by HRTEM of lower conductivity along the periodic direction than along the quasiperiodic directionof of the decagonal quasi-crystalline phase of the Al62 Cu20 Co15 Si3 alloy.
}

\author{
M. M. Martínez , R. García and J. Reyes- Gasga \\ Instituto de Física, Universidad Nacional Autónoma de México. Apartado Postal 20-364, México \\ D.F. Código Postal 01000, MÉXICO.
}

The discovery of a stable decagonal QC phase in $\mathrm{Al} \mathrm{Cu}$ Co by $\mathrm{He}$ et. al. [1] produced an importante research on these phases in the system $\mathrm{Al} \mathrm{Cu} \mathrm{Co}(\mathrm{Si})$. Moreover, QC phases obtained in this system were also reported with special electrical properties [2-4]. It is important to not, that these QC systems show a strong variation of the conductivity with a very slighhht change in composition. In agreement with differents authors, the electrical conductivity of the decagonal quasi-crystalls of the $\mathrm{Al} \mathrm{Cu} \mathrm{Co}(\mathrm{Si})$ alloy shows metallic behavior along to periodic direction and nonmetallic behavior along to quasiperiodic direction.

In this work we show some HRTEM results that indicate a different behavior of the electrical conductivity for decagonal phase af the $\mathrm{Al}_{62} \mathrm{Cu}_{20} \mathrm{Co}_{15} \mathrm{Si}_{3}$ alloy that was produced using a double elliptic mirror furnace with slow thermal inertial. We confirmed the decagonal structure of the $\mathrm{Al} \mathrm{Cu}$ Co Si grains by a tilting series of salected area diffraction patterns, Fig. 1. We obtained, that the quality of their atomic resolution electron microscope images is modify drastically with the composition of the alloy. Therfore, we find that the HRTEM images from our alloy were almost impossible to obtain because the lack of electrical conductivity of the sample produced too much astigmatism. The best images obtained are present in figure 2. Campare these with those obtained by Reyes-Gasga et al. [5] in a sample with different composition. In that case, this kind of aberration was not present.

The sample was ground and supported in cooper grids for the their trasmision electron microscope analysis. Electron difraction patterns, tilting experiments were obtained using a JEOL 100CX analytical electron microscope equipped with $\pm 60^{\circ}$ goniometer. Chemical analysis were carried out using a JEOL $2010 \mathrm{~F}$ analytical electron microscope equipped with a $\pm 22^{0}$ goniometer and a NORAN energy - dispersive X-ray analyzer For high resolution observation a JEOL 4000EX high resolution electron microcope was used.

\section{References}

[1] L. X. He, Y. K. Wu and K. H. Kuo, J. Mater. Sci Letters 7 (19988) 1284.

[2] Lin Shu- yuan, Wang Xue-mei, Lu Li and Zhang Dian-lin, Phys. Rev. B 41 (1990) 9625.

[3] S. Martin, A. F. Hebard, A. R. Kortan and F. A. Thiel, Phys. Rev. Lett. 67 (1991) 719.

[4] Wang Yun-ping, Zhang Dian-lin and L.F. Chen, Phys. Rev. B 48 (1993) 10542.

[5] J. Reyes-Gasga , A. Lara, H. Riveros, and M. José-Yacamán, Mat. Sci. Eng. A 150 (1992) 87.

[6] We thank from the technical help: Armando Lara, Roberto Hernández, José Guzmán, Luis Rendón, Manuel Aguilar, Jaqueline Cañetas, Carlos Flores, Samuel Tehuacanero, Gilberto Mondragón, Pedro Mexía, Carlos Magaña, Cristina Zorrilla and A. Sánchez. 

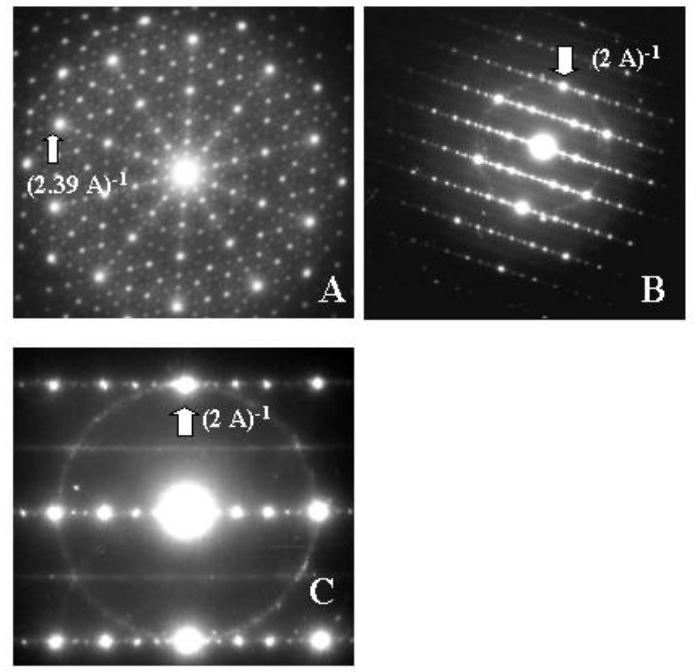

Fig. 1 EDP's of the decagonal Quasicrystalline phase. A) Along the 10-fold axis. B) - C) Along the two-fold axes, respectively.
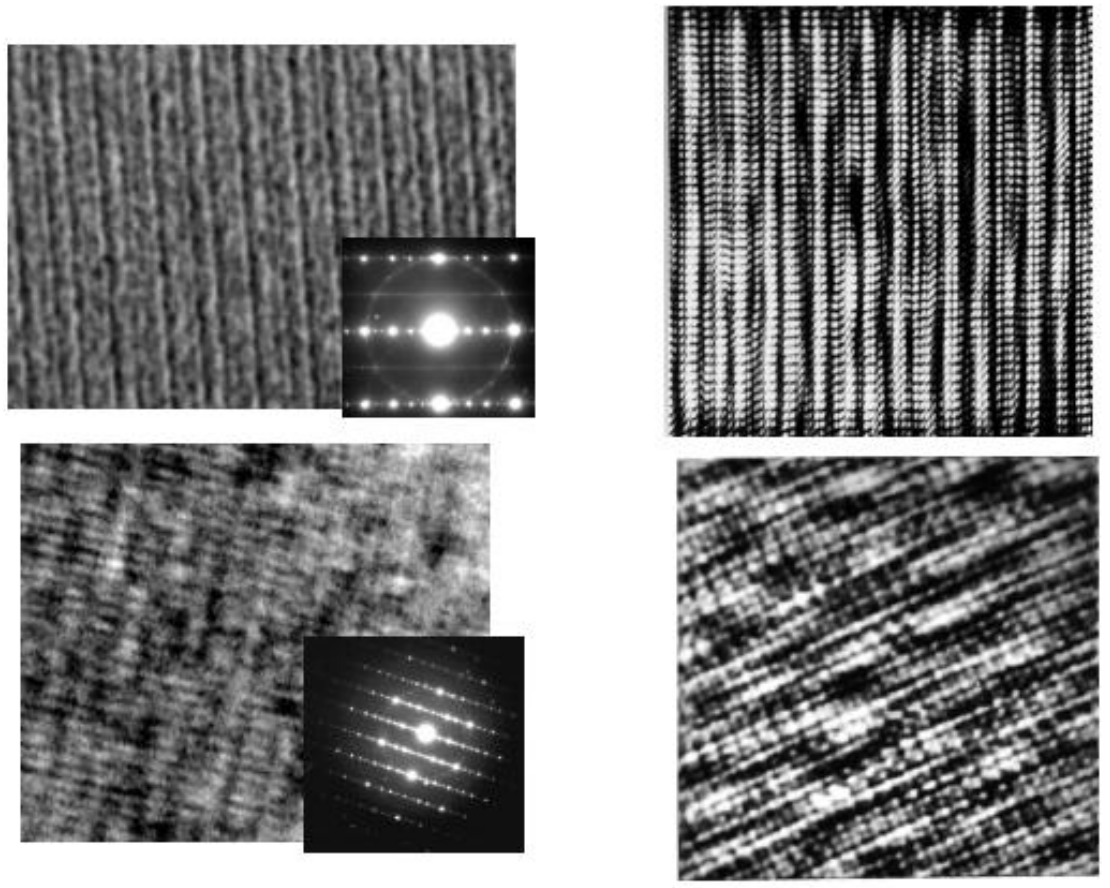

Fig. 2 Comparison between the high resolution images along the $\mathrm{D}$ and $\mathrm{P}$ axis, respectively, obtained from quasicrystals in this work and those obtained by J. Reyes-Gasga [6]. 
https://doi.org/10.1017/S1431927603443092 Published online by Cambridge University Press 